\title{
Upregulation of MAGEA4 correlates with poor prognosis in patients with early stage of esophageal squamous cell carcinoma
}

This article was published in the following Dove Press journal:

OncoTargets and Therapy

14 July 2016

Number of times this article has been viewed

\author{
Wei-Wei Tang' \\ Zi-Hao Liu \\ Tong-Xin Yang ${ }^{2}$ \\ Han-Jin Wang' \\ Xiu-Feng $\mathrm{Cao}^{2}$ \\ 'Department of General Surgery, \\ 2Department of Surgical Oncology, \\ Nanjing First Hospital, Nanjing \\ Medical University, Nanjing, Jiangsu, \\ People's Republic of China
}

Correspondence: Xiu-Feng Cao Department of Surgical Oncology, Nanjing First Hospital, Nanjing Medical University, 68 Changle Road, Nanjing 210006, Jiangsu, People's Republic of China

Tel +86 25 5227 I023

Fax +8625 52269924

Email cxf55। I01@sina.com

Han-Jin Wang

Department of General Surgery, Nanjing First Hospital, Nanjing Medical University, 68 Changle Road, Nanjing 210006, Jiangsu, People's Republic of China

Tel +86 25 52271023

Fax +862552269924

Emailwhj_888@sohu.com
Abstract: Esophageal cancer is a common type of cancer in the People's Republic of China. Many genes have been reported to be linked with it. Melanoma antigen gene family A ( $M A G E A)$ genes are frequently highly expressed in various types of carcinoma. However, the specific role of MAGEA gene expression in esophageal squamous cell carcinoma (ESCC) still remains unclear. MAGEA4 is a member of MAGEA genes. We aimed to investigate the expression and prognosis of MAGEA4 expression in ESCC. MAGEA4 messenger RNA expression levels of 120 pairs of tumor and nontumor tissues of patients with ESCC were measured by quantitative real-time polymerase chain reaction. The results showed that MAGEA4 messenger RNA was significantly elevated in tumor tissues of patients with ESCC compared to nontumor ones. In addition, overexpression of MAGEA4 messenger RNA was significantly correlated with poorer overall survival $(P=0.018)$ in early stage of patients with ESCC (I-IIA). In conclusion, MAGEA4 played an important role in the early stage of ESCC and overexpression of MAGEA4 was expected to become a potential prognostic marker for patients with early stage of ESCC.

Keywords: ESCC, metastasis, expression, survival, prognosis

\section{Introduction}

Esophageal cancer (EC) is the sixth common cause of cancer death worldwide and has become a major health concern, especially in Asia. ${ }^{1}$ There are two primary forms of EC, for example, esophageal squamous cell carcinoma (ESCC) and esophageal adenocarcinoma. ESCC is the most prevalent type in eastern countries, while esophageal adenocarcinoma often occurs in the West. Since the exact diagnosis is rarely made prior to advanced clinical stages, the overall 5-year survival rate of patients with ESCC remains extremely low, despite wide application of operation and chemo-radiotherapy. ${ }^{2-4} \mathrm{EC}$ ranks fourth in morbidity and mortality in People's Republic of China, after lung cancer, gastric cancer, and liver cancer. ${ }^{5}$ One of the main reasons for the low overall survival is the lack of appropriate molecular biomarkers for the early detection or prognosis of EC.

Melanoma antigen gene family A (MAGEA) family comprises 12 subtypes from MAGEA1 to MAGEA $12 .{ }^{6}$ Reports have shown that $M A G E A$ genes are upregulated in different types of cancer, such as lymphocytic leukemia, lung cancer, ovarian cancer, melanoma, and other cancers. ${ }^{7,8} M A G E A 4$ is a gene of $M A G E A$. Upregulation of $M A G E A 4$ has been found in several types of tumors, for example, oral squamous cell carcinoma, ${ }^{9}$ non-small-cell lung cancer, ${ }^{10}$ pancreatic cancer, ${ }^{11}$ and breast cancer. ${ }^{12}$ In the present study, we examined the expression of MAGEA4 in ESCC tissues and investigated the significance and prognostic value of $M A G E A$ expression in patients with ESCC. 


\section{Materials and methods}

\section{Patients and tissue samples}

A total of 120 ESCC and corresponding samples were obtained from patients who underwent surgery during 2001-2008 at Nanjing Hospital affiliated with the Nanjing Medical University, People's Republic of China. None of the patients received radiotherapy or chemotherapy before the operation. All specimens were immediately stored at $-80^{\circ} \mathrm{C}$ in a refrigerator until use. Each patient was followed up from 2 months to 5 years. The study was approved by the Ethics Committee of Nanjing First Hospital, Nanjing Medical University. Written informed consent was obtained from all patients before they participated in the research.

\section{RNA isolation and quantitative real-time polymerase chain reaction}

Total RNA was isolated from cancerous/noncancerous tissues with TRIzol reagent (Thermo Fisher Scientific, Waltham, MA, USA). Complementary DNAs were obtained with the PrimeScript $^{\mathrm{TM}}$ RT-PCR kit (Takara, Dalian, People's Republic of China). MAGEA4 messenger RNA (mRNA) expression was measured by quantitative real-time polymerase chain reaction (qRT-PCR) with the following primer sequences: forward, 5'-CCACTACCATCAGCTTCACTTGC-3' and reverse, 5'-AGGCAACCCAATGAGGGTTCCAGC-3'. The MAGEA4 mRNA levels were normalized to GAPDH using the sequences: forward, 5'-GTCAACGGATTTGGTCTGTATT-3' and reverse, 5'-AGTCTTCTGGGTGGCAGTGAT-3'. qRTPCR reactions were performed with ABI7500 System and SYBR Green PCR Master Mix (Thermo Fisher Scientific).

\section{Statistical analysis}

For ESCC tissues and the corresponding nontumor ones, the fold change of target gene is indicated by $2^{-\Delta \mathrm{CT}}$. All data were tested by Student's $t$-test, chi-square test, and analysis of variance, as appropriate. Overall survival curves were plotted by the method of Kaplan-Meier. Multivariate data were analyzed by Cox proportional hazards model. All statistical analyses were performed with Statistical Package for the Social Sciences Version 19 (IBM Corporation, Armonk, NY, USA). For all results, a $P$-value of $<0.05$ was considered statistically significant.

\section{Results}

\section{Expression of MAGEA4 mRNA in} patients with ESCC and normal tissues

The MAGEA4 expression levels in cancerous tissues and corresponding noncancerous tissues from 120 patients with

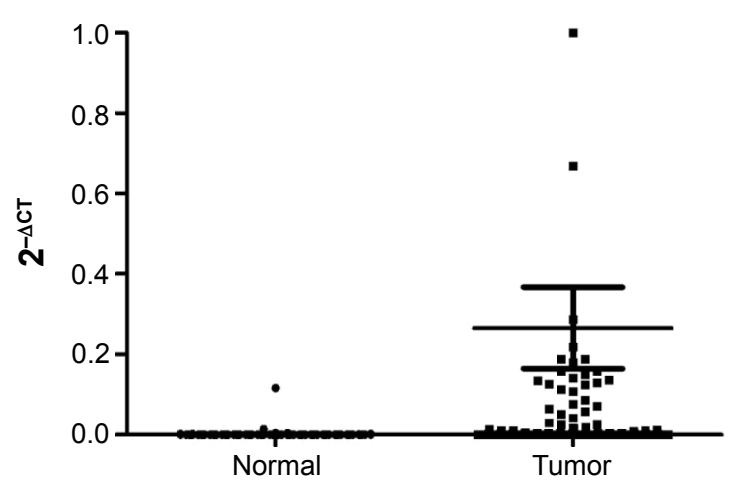

Figure I MAGEA4 expression in tumor tissues and normal tissues in 120 ESCC patients.

Abbreviations: ESCC, esophageal squamous cell carcinoma; MAGEA4, melanoma antigen gene family $A$.

ESCC were measured by qRT-PCR. Relative gene expression determinations were made with $2^{-\triangle \mathrm{CT}}$ method. MAGEA4 expression of tumor tissue was significantly higher than that of nontumor tissues (Figure 1).

\section{Clinical significance of MAGEA4 mRNA in patients with ESCC}

The association between MAGEA4 mRNA and clinicopathological features of 120 primary ESCC and nontumor samples was analyzed. As shown in Table 1, overexpression of MAGEA4 mRNA level was not aberrantly associated with age, sex, and differentiation in patients with ESCC. However, overexpression of MAGEA4 mRNA level was negatively associated with clinical stage and lymph node metastasis. The expression of MAGEA4 in patients with ESCC with I-IIA stage disease was higher than that in patients with IIB-IV stage disease. Patients with ESCC with lymph node metastasis showed lower MAGEA4 levels compared to those without lymph node metastasis.

\section{Upregulation of MAGEA4 in early stage of ESCC is linked to poor survival}

As the frequency of MAGEA4 overexpression was higher in early stages (I-IIA) than that in advanced stages (IIB-IV) (Table 1), the association of MAGEA4 overexpression with overall survival rate in patients with ESCC was studied subsequently. Kaplan-Meier analysis was used to examine the prognostic value of MAGEA4 mRNA for overall survival of patients with ESCC. Patients with ESCC with overexpression of MAGEA4 mRNA were significantly linked to poor overall survival (log rank $=5.565, P=0.018)$ (Figure 2). Univariate analysis showed that tumor node metastasis (TNM) stage $(P<0.05)$, MAGEA4 overexpression $(P=0.032)$, and lymph node metastasis $(P<0.05)$ were independent 
Table I Clinicopathological characteristics and expression of MAGEA4

\begin{tabular}{|c|c|c|c|c|}
\hline \multirow{2}{*}{$\begin{array}{l}\text { Clinicopathological } \\
\text { characteristics }\end{array}$} & \multirow[t]{2}{*}{ Number (n) } & \multicolumn{2}{|l|}{ MAGEA4 mRNA (n) } & \multirow[t]{2}{*}{$P$-value } \\
\hline & & Without overexpression (\%) & With overexpression (\%) & \\
\hline Sex & & 13 & 107 & 0.071 \\
\hline Male & 98 & $13(13.3)$ & $85(86.7)$ & \\
\hline Female & 22 & $0(0.0)$ & $22(100.0)$ & \\
\hline Age & & & & 0.204 \\
\hline$\geq 62$ years & 57 & $4(7.0)$ & $53(93.0)$ & \\
\hline$<62$ years & 63 & $9(14.3)$ & $54(85.7)$ & \\
\hline Histological type & & & & 0.362 \\
\hline High-middle & 100 & $12(12.0)$ & $88(88.0)$ & \\
\hline Low & 20 & I (5.0) & $19(95.0)$ & \\
\hline TNM stage group & & & & $0.001 *$ \\
\hline I-IIA & 85 & $9(10.1)$ & $76(89.4)$ & \\
\hline IIB-IV & 35 & $4(11.4)$ & $31(88.6)$ & \\
\hline Lymph node metastasis & & & & $0.001 *$ \\
\hline Yes & 35 & $9(25.7)$ & $26(74.3)$ & \\
\hline No & 85 & $4(4.7)$ & $81(95.3)$ & \\
\hline
\end{tabular}

Note: $* P<0.05$

Abbreviations: MAGEA4, melanoma antigen gene family A 4; TNM, tumor node metastasis; mRNA, messenger RNA.

prognostic factors for the 120 informative ESCC samples (Table 2).

\section{Discussion}

A lack of efficient ESCC therapy makes it urgent to identify specific biomarkers as potential therapeutic targets of patients with ESCC. The method of qRT-PCR has made it possible to detect molecular biomarkers for the assessment

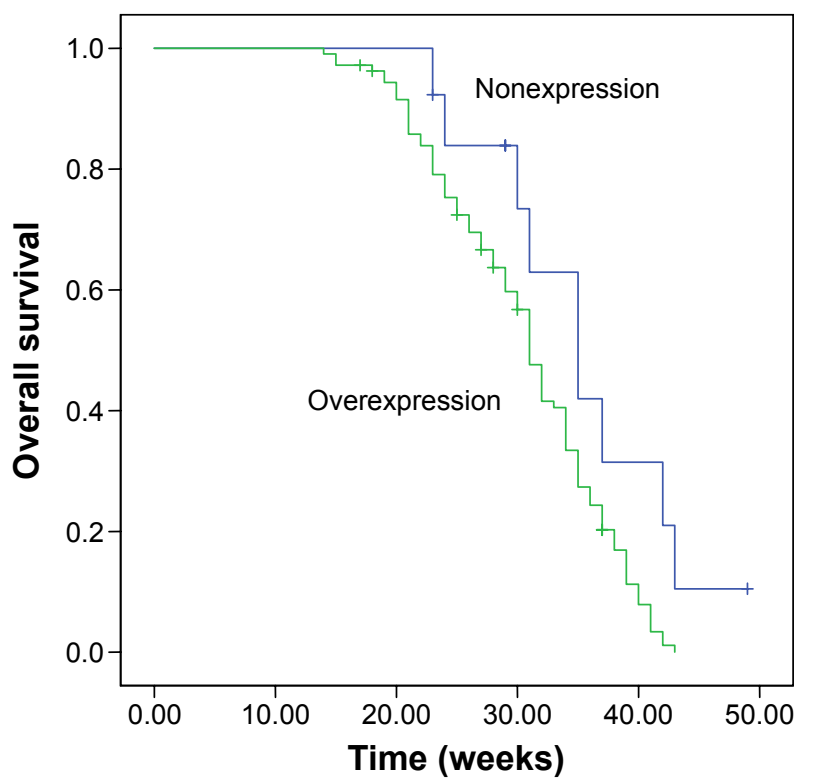

Figure 2 Prognostic value of MAGEA4 mRNA for overall survival of patients with ESCC in Kaplan-Meier analysis.

Abbreviations: ESCC, esophageal squamous cell carcinoma; MAGEA4, melanoma antigen gene family $A$ 4; mRNA, messenger RNA. of micrometastasis..$^{13}$ MAGEA4 is a member of the cancer/ testis antigen family and does not express in normal tissues except in the placenta or testis. The expression of cancer testis antigen is most specific for cancer. MAGEA4 expresses in several carcinomas, for example, head and neck cancer (53\%), lung cancer (51\%), bladder cancer (33\%), and cancer of esophagus (63\%). ${ }^{14}$ MAGEA4 is expected to be the optimal candidate for being a therapeutic target of ESCC.

To our knowledge, our study is the first one to detect MAGEA4 mRNA expression in tissues of patients with ESCC. Chaux et al ${ }^{14}$ found that MAGEA4 is a member of the cancer/testis antigen family and does not express in normal tissues except in the placenta or testis. Sharma et al ${ }^{15}$ examined the expression of seven gene products including MAGEA4 in 94 bladder tumor samples and observed that MAGEA4 had the highest incidence of expression compared to other types of genes. As a result, MAGEA4 is expected to be the optimal candidate for being a therapeutic target of ESCC.

In the present study, we examined the expression of MAGEA4 genes in ESCC tissues and their corresponding nontumor esophageal tissues. Our results demonstrated that in 120 pairs of tumor and nontumor esophageal tissues, MAGEA4 expression of tumor tissues was significantly higher than that of nontumor tissues. We analyzed its relationship with the clinical data and found that MAGEA4 mRNA level was not linked to age, sex, and differentiation in ESCC. However, overexpression of MAGEA4 mRNA level was negatively associated with clinical stage and lymph 
Table 2 Univariate and multivariate Cox analyses

\begin{tabular}{|c|c|c|c|}
\hline Variables & HR & $95 \% \mathrm{Cl}$ & $P$-value \\
\hline \multicolumn{4}{|l|}{ Univariate Cox analysis } \\
\hline Sex (male vs female) & 1.166 & $0.716-1.899$ & 0.537 \\
\hline TNM stage (I-IIA vs IIB-IV) & 2.838 & I.79I-4.498 & $0.000 *$ \\
\hline MAGEA4 overexpression (without vs with) & 2.165 & $1.068-4.388$ & $0.032 *$ \\
\hline Lymph node metastasis (without vs with) & 2.838 & I.79I-4.498 & $0.000 *$ \\
\hline \multicolumn{4}{|l|}{ Multivariate Cox analysis } \\
\hline Sex (male vs female) & 1.357 & $0.807-2.281$ & 0.250 \\
\hline Age ( $\geq 62$ years vs $<62$ years $)$ & 1.124 & $0.74 I-I .704$ & 0.583 \\
\hline TNM stage (I-IIA vs IIB-IV) & 3.778 & $2.335-6.114$ & $0.000^{*}$ \\
\hline MAGEA4 overexpression (without vs with) & 3.385 & $1.634-7.014$ & $0.001 *$ \\
\hline Histological type (high-middle vs low) & 0.923 & $0.548-1.554$ & 0.763 \\
\hline Lymph node metastasis (without vs with) & 3.778 & $2.335-6.114$ & $0.000 *$ \\
\hline
\end{tabular}

Note: $* p<0.05$.

Abbreviations: $\mathrm{Cl}$, confidence interval; $\mathrm{HR}$, hazard ratio; MAGEA4, melanoma antigen gene family A 4; TNM, tumor node metastasis.

node metastasis. Clinical stage and lymph node metastasis are two important indicators of tumor malignancy of patients with ESCC. Patients with ESCC with overexpressed MAGEA4 are aberrantly associated with poorer overall survival, compared to patients not showing overexpression of MAGEA4 in tumors. Univariate Cox proportional hazard regression analysis showed that MAGEA4 mRNA was not an independent factor for overall survival. Sex, TNM stage, and lymph node metastasis are all dangerous factors for overall survival of patients with ESCC.

In the present study, we examined the expression of MAGEA4 genes in ESCC tissues and their corresponding nontumor esophageal tissues. Our results demonstrated that in 120 pairs of tumor and nontumor esophageal tissues, MAGEA4 expression of tumor tissue was significantly higher than that of nontumor tissues. This trend is consistent with other reports. ${ }^{14}$ ESCC patients with higher MAGEA4 mRNA expression are more prone to lymph node metastasis. Kaplan-Meier analysis showed that patients with ESCC with overexpression of MAGEA4 mRNA were significantly linked to poor overall survival. Figure 2 showed the prognostic value of MAGEA4 mRNA for overall survival of patients with ESCC, indicating that the higher the expression, the worse the prognosis.

Patients carrying ESCC with overexpressed MAGEA4 are aberrantly associated with poorer overall survival, compared to patients not showing overexpression of MAGEA4 in tumors. In our research, we did a comprehensive analysis of several factors by multivariate Cox proportional hazard regression analysis and found that only three factors were meaningful. They were TNM stage, lymph node metastasis, and MAGEA4 mRNA expression. Sex, age, and differentiation may not influence the overall survival of patients with ESCC independently.

\section{Conclusion}

In summary, our data demonstrated that MAGEA4 mRNA was commonly upregulated in patients with ESCC. Overexpression of MAGEA4 mRNA in ESCC tumor was significantly associated with poor overall survival of patients with ESCC. Therefore, MAGEA4 mRNA could be used as a prognostic marker for predicting the overall survival of patients with ESCC after surgery.

\section{Acknowledgment}

This study was supported by the National Natural Science Foundation of China (grant no H1617/81201881).

\section{Disclosure}

The authors report no conflicts of interest in this work.

\section{References}

1. Jemal A, Bray F, Center MM, Ferlay J, Ward E, Forman D. Global cancer statistics. CA Cancer J Clin. 2011;61(2):69-90.

2. Pohl H, Welch HG. The role of overdiagnosis and reclassification in the marked increase of esophageal adenocarcinoma incidence. J Natl Cancer Inst. 2005;97(2):142-146.

3. Isono K, Sato H, Nakayama K. Results of a nationwide study on the three field lymph node dissection of esophageal cancer. Oncology. 1991; 48(5):411-420.

4. Pisani P, Parkin DM, Bray F, Ferlay J. Estimates of the worldwide mortality from 25 cancers in 1990. Int J Cancer. 1999;83(1):18-29.

5. Chen W, Zheng R, Zeng H, Zhang S, He J. Annual report on status of cancer in China, 2011. Chin J Cancer Res. 2015;27(1):2-12.

6. Zammatteo N, Lockman L, Brasseur F, et al. DNA microarray to monitor the expression of MAGE-A genes. Clin Chem. 2002;48(1):25-34.

7. Park JW, Kwon TK, Kim IH, et al. A new strategy for the diagnosis of MAGE-expressing cancers. J Immunol Methods. 2002;266(1-2): 79-86.

8. De Plaen E, Arden K, Traversari C, et al. Structure, chromosomal localization, and expression of 12 genes of the MAGE family. Immunogenetics. 1994;40(5):360-369.

9. Montoro JR, Mamede RC, Neder Serafini L, et al. Expression of cancer-testis antigens MAGE-A4 and MAGE-C1 in oral squamous cell carcinoma. Head Neck. 2012;34(8):1123-1128. 
10. Baba T, Shiota H, Kuroda K, et al. Clinical significance of human leukocyte antigen loss and melanoma-associated antigen 4 expression in smokers of non-small cell lung cancer patients. Int J Clin Oncol. 2013;18(6):997-1004. Epub 2012 Nov 3.

11. Kim J, Reber HA, Hines OJ, et al. The clinical significance of MAGEA3 expression in pancreatic cancer. Int J Cancer. 2006;118(9): 2269-2275.

12. Wong PP, Yeoh CC, Ahmad AS, et al. Identification of MAGEA antigens as causal players in the development of tamoxifen-resistant breast cancer. Oncogene. 2014;33(37):457988.
13. Daniele B, Bencivenga A, Megna AS, Tinessa V. Alpha-fetoprotein and ultrasonography screening for hepatocellular carcinoma. Gastroenterology. 2004;127(5 Suppl 1):S108-S112.

14. Chaux P, Luiten R, Demotte N, et al. Identification of five MAGE-A1 epitopes recognized by cytolytic $\mathrm{T}$ lymphocytes obtained by in vitro stimulation with dendritic cells transduced with MAGE-A1. J Immunol. 1999;163(5):2928-2936.

15. Sharma P, Shen Y, Wen S, et al. Cancer-testis antigens: expression and correlation with survival in human urothelial carcinoma. Clin Cancer Res. 2006;12(18):5442-5447.

\section{Publish your work in this journal}

OncoTargets and Therapy is an international, peer-reviewed, open access journal focusing on the pathological basis of all cancers, potential targets for therapy and treatment protocols employed to improve the management of cancer patients. The journal also focuses on the impact of management programs and new therapeutic agents and protocols on

\section{Dovepress}

patient perspectives such as quality of life, adherence and satisfaction. The manuscript management system is completely online and includes a very quick and fair peer-review system, which is all easy to use. Visit http://www.dovepress.com/testimonials.php to read real quotes from published authors. 